\title{
Tunable discrete scale invariance in transition-metal pentatelluride flakes
}

Yanzhao Liu ${ }^{1,11}$, Huichao Wang ${ }^{2,11}$, Haipeng $\mathrm{Zhu}^{3}$, Yanan $\mathrm{Li}^{1}$, Jun Ge${ }^{1}$, Junfeng Wang ${ }^{3}$, Liang $\mathrm{Li}^{3}$, Ji-Yan Dai ${ }^{4}$, Jiaqiang Yan ${ }^{5}$, David Mandrus ${ }^{5,6}$, Robert Joynt ${ }^{7,8}$ and Jian Wang $\mathbb{D}^{1,9,10 \bowtie}$

Log-periodic quantum oscillations discovered in transition-metal pentatelluride give a clear demonstration of discrete scale invariance (DSI) in solid-state materials. The peculiar phenomenon is convincingly interpreted as the presence of two-body quasibound states in a Coulomb potential. However, the modifications of the Coulomb interactions in many-body systems having a Dirac-like spectrum are not fully understood. Here, we report the observation of tunable log-periodic oscillations and DSI in ZrTe ${ }_{5}$ and $\mathrm{HfTe}_{5}$ flakes. By reducing the flakes thickness, the characteristic scale factor is tuned to a much smaller value due to the reduction of the vacuum polarization effect. The decreasing of the scale factor demonstrates the many-body effect on the DSI, which has rarely been discussed hitherto. Furthermore, the cut-offs of oscillations are quantitatively explained by considering the Thomas-Fermi screening effect. Our work clarifies the many-body effect on DSI and paves a way to tune the DSI in quantum materials.

npj Quantum Materials (2020)5:88; https://doi.org/10.1038/s41535-020-00290-6

\section{INTRODUCTION}

One of the most important concepts in the area of phase transitions is scale invariance. A scale invariant system reproduces itself on different temporal and spatial scales. This is described by the relation $f(k x)=k^{\lambda} f(x)$, where $k$ is an arbitrary parameter, $\lambda$ is the scaling dimension, and $f$ is a physical field. Discrete scale invariance (DSI) is a weaker case of the scale invariance, where a system only obeys the scale invariance for specific choices of $k^{1,2}$. With a fundamental scaling ratio $\lambda$ and characteristic logperiodicity, DSI arises in various contexts, such as earthquakes, financial crashes, turbulence and so on ${ }^{1}$. After being introduced to bound-state problems of quantum systems by Vitaly Efimov in 1970 (ref. ${ }^{3}$ ), the DSI had been observed only in cold atom systems for a long time ${ }^{4-7}$. It had not previously been observed in the solid state. The discovery of Dirac materials has changed that situation $^{8-14}$. Especially in the topological transition-metal pentatelluride $\mathrm{ZrTe}_{5}$, the quantum oscillations with log-periodicity have revealed the existence of DSI in a solid-state system ${ }^{10}$. The origin is attributed to the quasi-bound states induced by the supercritical Coulomb interaction between the massless Dirac fermions and charged impurities ${ }^{10,12,13}$. Further studies reported the logperiodic oscillations in $\mathrm{HfTe}_{5}{ }^{14}$ and elemental semiconductor tellurium ${ }^{15}$, confirming that the DSI feature can be a universal characteristic of Dirac materials with Coulomb impurities.

$\mathrm{ZrTe}_{5}$ and $\mathrm{HfTe}_{5}$ are predicted to be quantum spin Hall insulators in the two-dimensional (2D) limit and the threedimensional (3D) crystals are located near the phase boundary between weak and strong topological insulators ${ }^{16}$. Later studies indicated that the topological natures of both $\mathrm{ZrTe}_{5}$ and $\mathrm{HfTe}_{5}$ are very sensitive to the crystal lattice constant and detailed composition $^{17,18}$. Taking $\mathrm{ZrTe}_{5}$ as an example, some angleresolved photoemission spectroscopy (ARPES) ${ }^{19,20}$ and magnetoinfrared spectroscopy studies show that $\mathrm{ZrTe}_{5}$ is a Dirac semimetal $^{21}$. The observed negative magnetoresistance (MR) that is related to the chiral anomaly and the anomalous Hall effect support the hypothesis of a massless Dirac band structure ${ }^{22-24}$. However, other ARPES and scanning tunneling microscopy (STM) results suggest that $\mathrm{ZrTe}_{5}$ is a topological insulator ${ }^{25-28}$. Thus, the transition-metal pentatelluride $\mathrm{ZrTe}_{5}$ and $\mathrm{HfTe}_{5}$ are ideal platforms to investigate different intriguing physical properties due to their high tunability ${ }^{29-34}$. In particular, the very small Fermi surface of the compounds has enabled some peculiar findings in the ultraquantum regime, such as log-periodic quantum oscillations and $3 D$ quantum Hall effect ${ }^{10,14,35}$. As described above, the logperiodic oscillations revealing the DSI feature can be convincingly explained by a two-body quasi-bound state model. However, the solids $\mathrm{ZrTe}_{5}$ and $\mathrm{HfTe}_{5}$ are in fact many-body systems, and the Coulomb interactions are modified by screening effects that are not fully understood. The screening of the Coulomb interaction is closely related to the carrier density. In the experiments, we find that the carrier density in $\mathrm{ZrTe}_{5}$ flakes changes with thickness ${ }^{29}$. The interaction between the layers of $\mathrm{ZrTe}_{5}$ and $\mathrm{HfTe}_{5}$ is comparable to graphene, making it easy to get flakes from the bulk samples by exfoliation ${ }^{16}$. Thus, it is interesting to study the log-periodic quantum oscillations in the transition-metal pentatelluride flakes with different carrier density by thickness control, which may provide insights into many-body effects on DSI.

In this work, we carried out systematic magnetotransport measurements on $\mathrm{ZrTe}_{5}$ and $\mathrm{HfTe}_{5}$ flakes under magnetic fields $(B)$ up to 53 Tesla $(T)$. The $\log B$-periodic oscillations can be detected in both $\mathrm{ZrTe}_{5}$ and $\mathrm{HfTe}_{5}$ flakes with thicknesses down to about

\footnotetext{
${ }^{1}$ International Center for Quantum Materials, School of Physics, Peking University, 100871 Beijing, China. ${ }^{2}$ School of Physics, Sun Yat-Sen University, 510275 Guangzhou, China. ${ }^{3}$ Wuhan National High Magnetic Field Center, Huazhong University of Science and Technology, 430074 Wuhan, China. ${ }^{4}$ Department of Applied Physics, The Hong Kong Polytechnic University, Kowloon, Hong Kong, China. ${ }^{5}$ Materials Science and Technology Division, Oak Ridge National Laboratory, Oak Ridge, TN 37831, USA. ${ }^{6}$ Department of Materials Science and Engineering, University of Tennessee, Knoxville, TN 37996, USA. ${ }^{7}$ Kavli Institute of Theoretical Sciences, Chinese Academy of Sciences, 100049 Beijing, China. ${ }^{8}$ Department of Physics, University of

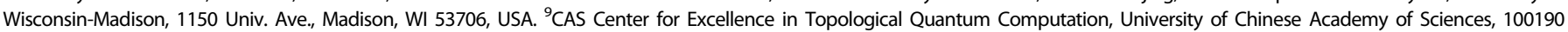
Beijing, China. ${ }^{10}$ Beijing Academy of Quantum Information Sciences, 100193 Beijing, China. ${ }^{11}$ These authors contributed equally: Yanzhao Liu and Huichao Wang. email: jianwangphysics@pku.edu.cn
} 
a

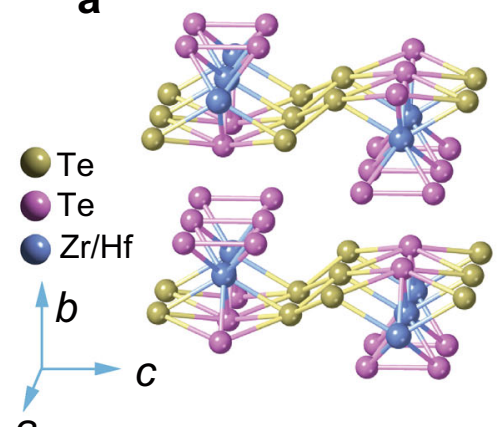

a b

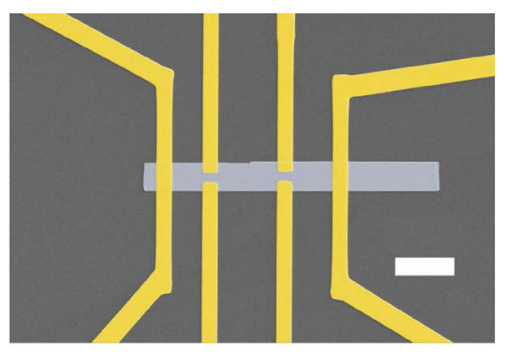

C

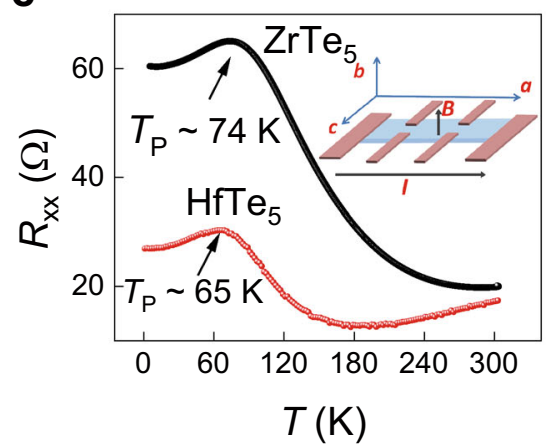

Fig. 1 Crystal structure and resistance-temperature characteristics of the transition-metal pentatelluride flakes. a Crystal structures of $\mathrm{ZrTe}_{5}$ and $\mathrm{HfTe}_{5}$. b Scanning electron microscope false color image of a typical ZrTe $e_{5}$ flake. Scale bar represents $10 \mu \mathrm{m}$. c Typical temperaturedependent longitudinal resistance of the transition-metal pentatelluride flakes. The peak anomaly appears at $T_{\mathrm{p}} \sim 65 \mathrm{~K}$ and $74 \mathrm{~K}$ for $\mathrm{HfTe}{ }_{5}$ $\left(300 \mathrm{~nm}\right.$ thick) and $\mathrm{ZrTe}_{5}(190 \mathrm{~nm}$ thick) flakes, respectively. Inset shows a schematic structure for the electrical transport measurements.

$160 \mathrm{~nm}$. In the thick $\mathrm{ZrTe}_{5}$ flake $(\sim 1200 \mathrm{~nm}), \log B$ oscillations with a scale factor $\lambda \sim 3.3$ is observed. The consistent scale factors between the flake $(\lambda \sim 3.3)$ and bulk crystals $(\lambda \sim 3.2)^{10}$ show that the quality of the samples is not affected by the process of exfoliation and flake fabrication. More interestingly, a relatively smaller scale factor $\lambda \sim 1.6$ can be extracted in the MR and Hall traces of flakes with a thickness of about $160 \mathrm{~nm}$. Theoretically, this index $\lambda$ decreases with increasing the effective charge of impurities that compose quasi-bound states responsible for the DSI and the log-periodicity. Further analysis shows that the carrier density in the flakes increases as the thickness decreases. We believe that the high carrier density makes the vacuum polarization effect weaker and leads to a larger effective charge, which results in a smaller scale factor. Thus, this work reveals a thickness-tuned scale factor of $\log B$ quantum oscillations in $\mathrm{ZrTe}_{5}$ and $\mathrm{HfTe}_{5}$ flakes and provides a perspective on the DSI in solidstate systems.

\section{RESULTS}

Temperature dependence of resistance

$\mathrm{ZrTe}_{5}$ and $\mathrm{HfTe}_{5}$ belong to the orthorhombic space group $\mathrm{Cmcm}$ $\left(D_{2 h}^{17}\right)^{36}$. Figure 1a shows the crystal structure of $\mathrm{ZrTe}_{5}$ and $\mathrm{HfTe}_{5}$. Within the $a-c$ plane, the trigonal prismatic chains of " $\mathrm{ZrTe}_{3}$ " or " $\mathrm{HfTe}_{3}$ " run along the $a$ axis and are linked by parallel zigzag chains of " $\mathrm{Te}_{2}$ " along the $c$ axis. The layers of $\mathrm{ZrTe}_{5}$ and $\mathrm{HfTe}_{5}$ are stacked along the $b$ axis. The false-color scanning electron microscopy (SEM) image of a fabricated $\mathrm{ZrTe}_{5}$ flake (gray color) with six electrodes (gold color) is displayed in Fig. 1b. A schematic measurement structure of standard six-electrode-method is illustrated in the inset of Fig. 1c. The current is applied along the $a$ axis and the magnetic field is along the $b$ axis for all measurements.

Figure 1c represents typical resistance-temperature $(R-T)$ curves of $\mathrm{HfTe}_{5}\left(\sim 300 \mathrm{~nm}\right.$ thick) and $\mathrm{ZrTe}_{5}(\sim 190 \mathrm{~nm}$ thick) flakes. Resistance peaks at $T_{\mathrm{p}} \sim 65 \mathrm{~K}$ and $74 \mathrm{~K}$ can be detected for $\mathrm{HfTe}_{5}$ and $\mathrm{ZrTe}_{5}$ flakes, respectively. It is noted that the $T_{\mathrm{p}}$ of the flakes are definitely higher than that of the bulk ${ }^{10,14}$. The origin of the resistance peak in $\mathrm{ZrTe}_{5}$ and $\mathrm{HfTe}_{5}$ has been discussed for decades, and recently the view of the Lifshitz transition during the changing of temperatures explains some findings in the compounds ${ }^{28}$. However, in other reports, the Hall resistances of $\mathrm{ZrTe}_{5}$ and $\mathrm{HfTe}_{5}$ remain $p$ type with increasing temperatures, which is not consistent with the picture of a Lifshitz transition ${ }^{29,37}$. An alternative two-band model has been presented to be responsible for the transport anomaly and the various $T_{\mathrm{p}}$ in different samples ${ }^{14,29}$. In this picture, at low temperatures, the metallic $R-T$ curve mainly relies on a semi-metallic Dirac band, while a semiconducting band dominates at higher temperatures. The combination and competition between the two bands lead to the resistance peak at $T_{\mathrm{p}}$. In exfoliated flakes, the carrier density of the Dirac holes commonly increases with decreasing thickness, indicating a shift of the Fermi level toward the valence band. Thus, the thickness decrease can alter the competition balance and result in a higher $T_{\mathrm{p}}$.

Tunable log-periodic oscillations and DSI

A $\mathrm{ZrTe}_{5}$ flake labeled s1 with a thickness of about $1200 \mathrm{~nm}$ was measured under magnetic fields up to $53 \mathrm{~T}$ and the MR curves in a semilogarithmic scale are shown in Fig. 2a. By subtracting a smooth background, the $\log B$-periodic oscillating components can be extracted and are shown in Fig. 2b. For clarity, data curves in Fig. 2a, b are shifted. Distinct log-periodic magneto-oscillations can be observed at low temperatures. With increasing temperature, thermal broadening eventually becomes comparable to the intrinsic width of the quasi-bound states that induce log-periodic oscillations ${ }^{10,14}$. Thus, the oscillations gradually attenuate with increasing temperature and finally disappear above $120 \mathrm{~K}$ (see Supplementary Fig. 1a, b), which agree with the observations in bulk crystals ${ }^{10}$. Consistent results were also acquired by using the second differential method to extract the oscillations, as shown in Supplementary Fig. 1d. The independence of the oscillations on the subtraction methods demonstrates that the log-periodic structures are intrinsic properties of the materials (see Supplementary Note 1 and Supplementary Figs. 1-5 for more details). The characteristic magnetic fields $\left(B_{n}\right)$ of the observed oscillation peaks and dips can be indexed as $n$ and $(n-0.5)$, respectively. By plotting $\log \left(B_{n}\right)$ as a function of $n$, a linear dependence is revealed to confirm the log-periodic property (Fig. 2C). Based on the linear fitting in Fig. 2c, a dominant scale factor $\lambda=B_{n} / B_{n+1} \sim 3.34$ can be obtained. Further, the Fast Fourier Transform (FFT) result of the log-periodic oscillations is shown in Fig. 2d. The sharp FFT frequency peak is located at $F \sim 1.87$, which indicates a period of $\log \left(B_{n}\right) \sim 0.53$ and a main scale factor $\lambda \sim 3.38$. The FFT shows some broadening, with a full-width at half-maximum (FWHM) analysis giving a reasonable $\lambda$ range of about $[2.58,5.98]$. It is noted that the properties of the oscillations in the 1200-nm-thick $\mathrm{ZrTe}_{5}$ flake are very close to those observed in the bulk ${ }^{10}$, showing that the quality of the device is still reserved during the process of the thin flake fabrication.

The thinner flakes are further studied for comparison. Figure 3 shows the log-periodic oscillations in the MR and Hall traces of the $\mathrm{ZrTe}_{5}$ flake (s2) with a thickness of about $137 \mathrm{~nm}$. The oscillatory part of MR at $4.2 \mathrm{~K}$ after subtracting a smooth background is displayed in Fig. 3a. The inset of Fig. 3a plots the MR of $s 2$ at $4.2 \mathrm{~K}$ as a function of the magnetic field. The log-periodic oscillations can be observed and 

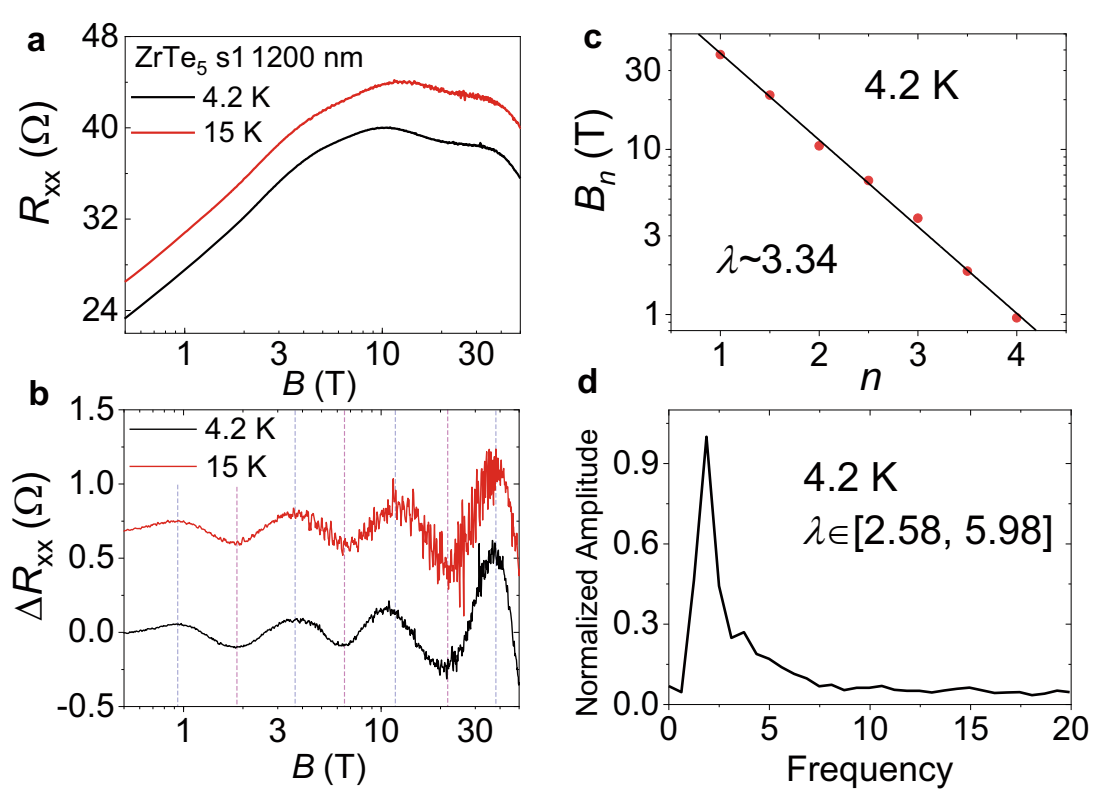

Fig. 2 Log-periodic MR oscillations and DSI in the $\mathrm{ZrTe}_{5}$ flake (s1) with a thickness of about $1200 \mathrm{~nm}$. a MR of s1 at $4.2 \mathrm{~K}$ and $15 \mathrm{~K}$ versus magnetic field. b Extracted MR oscillatory part from the data in a. c LogB-periodicity of the MR oscillations in s1. d FFT result of the MR oscillations at $4.2 \mathrm{~K}$ in $\mathbf{b}$.

a

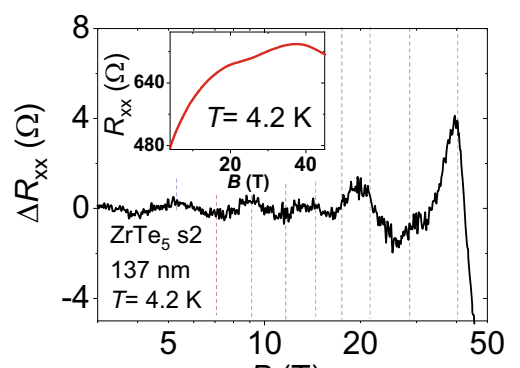

b

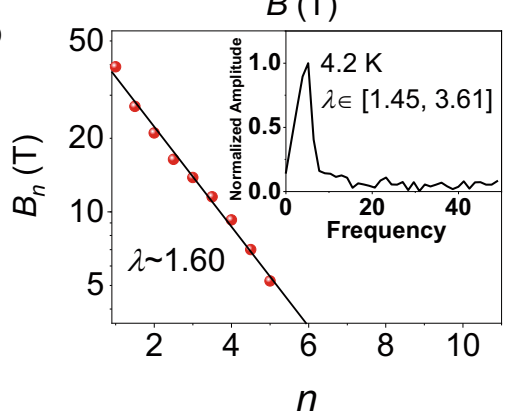

c

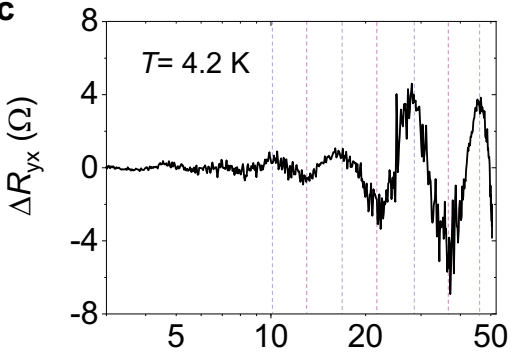

d $\quad B(T)$

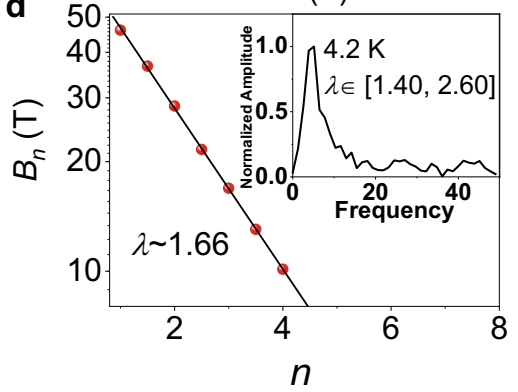

Fig. 3 Log-periodic oscillations and DSI in the $\mathrm{ZrTe}_{5}$ flake (s2) with a thickness of about $137 \mathrm{~nm}$. a Extracted oscillatory part from MR of s2 at $4.2 \mathrm{~K}$. Inset: MR of s2 at $4.2 \mathrm{~K}$. b LogB-periodicity of the MR oscillations in s2. Inset: FFT result of the MR oscillations at $4.2 \mathrm{~K}$ in a. The scale factor $\lambda$ of the oscillations in the MR is about 1.60. $\mathbf{c}$, d Results for the Hall trace of $s 2$ at $4.2 \mathrm{~K}$. The scale factor $\lambda$ of the oscillations in the Hall trace is about 1.66 , coincided with that for the MR.

survive up to $120 \mathrm{~K}$ (Supplementary Fig. 6). The index plot and FFT result of the oscillations are shown in Fig. 3b. Both the linear fitting and the sharp FFT frequency peak confirm the log-periodicity and give a $\lambda \sim 1.60$. The FWHM of the frequency peak further indicates a range for $\lambda$ of $[1.45,3.61]$. The log-periodic oscillations are also observed in the Hall traces of the s2, as shown in Fig. 3c, d. We subtract the background of the Hall signal by using similar methods to the MR. $\Delta R_{y x}$ as a function of $\log B$ is plotted in Fig. 3c. Figure $3 d$ displays the linear fitting of the index and the FFT analysis of the data shown in Fig. 3c. A scale factor $\lambda \sim 1.66$ can be obtained for the oscillations in the Hall resistance, coinciding with $\lambda \sim 1.60$ for the MR.
The near equality of $\lambda$ in the MR and the Hall resistance is in agreement with the theoretical expectation ${ }^{14}$. Further measurements on a $\mathrm{ZrTe}_{5}$ flake with a thickness of about $50 \mathrm{~nm}$ show log-periodic quantum oscillations in Hall trace with $\lambda \sim 1.52$ (Supplementary Fig. 7). The decrease of the scale factor is also supported by the observations in the $\mathrm{HfTe}_{5}$ flake, which shows clearer oscillations. Figure 4 displays the magnetotransport results of the $\mathrm{HfTe}_{5}$ flake (s3) with a thickness of about $180 \mathrm{~nm}$. By using the same background subtraction method, the log-periodic oscillations in MR and Hall traces can be extracted, as shown in Fig. $4 \mathrm{a}$, c. The oscillations in MR at high magnetic fields can survive up to $78 \mathrm{~K}$ (Supplementary Fig. 8). Figure $4 \mathrm{~b}$, d plots the linear 

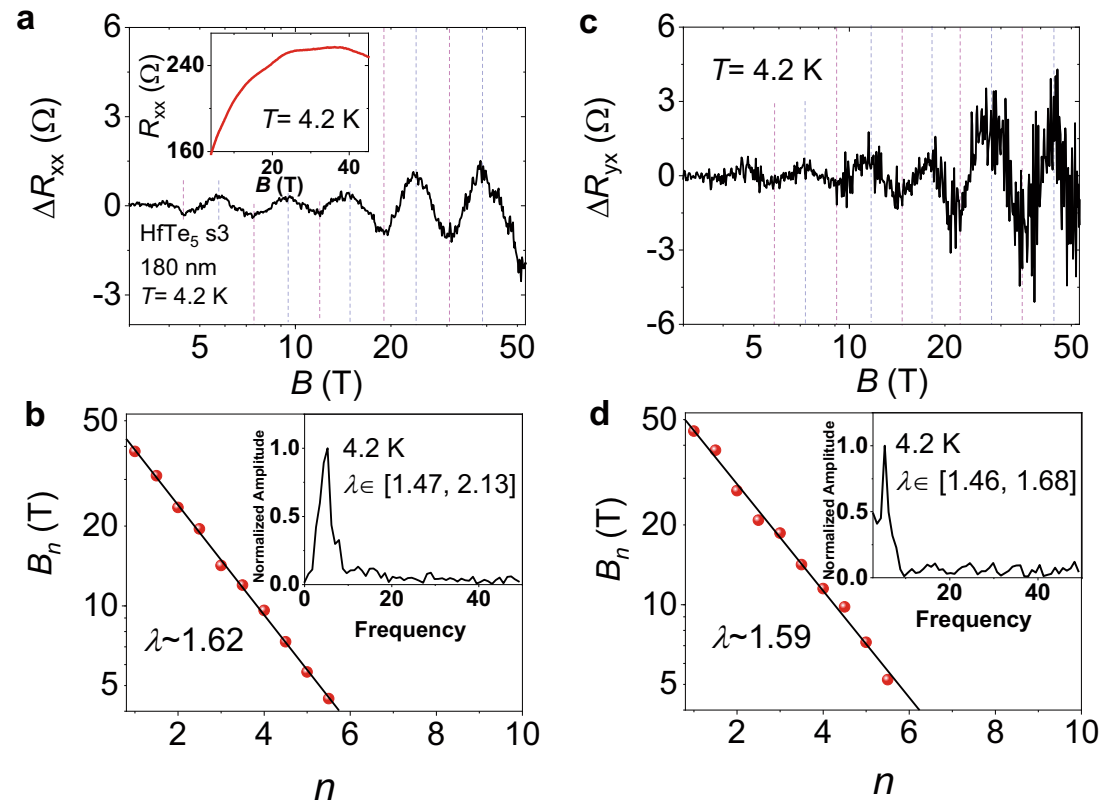

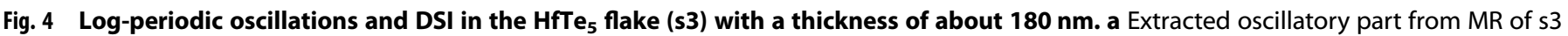
at $4.2 \mathrm{~K}$. Inset: MR of s3 at $4.2 \mathrm{~K}$. b LogB-periodicity of the MR oscillations in s3. Inset: FFT result of the MR oscillations at $4.2 \mathrm{~K}$ in a. c, $\mathbf{d}$ Results for the Hall traces of $s 3$ at $4.2 \mathrm{~K}$. The scale factor $\lambda$ for the log-periodic oscillations in the Hall trace is about 1.59, consistent with that ( 1.62) for the MR.

fitting and FFT analysis for the oscillations in the MR and the Hall trace, respectively. The scale factor $\lambda$ in $\mathrm{s} 3$ is estimated to be around 1.6 , smaller than $\lambda \sim 3.5$ in the bulk ${ }^{14}$.

\section{DISCUSSION}

As discussed in previous works, the log-periodic quantum oscillations can be attributed to the two-body quasi-bound states composed of a massless Dirac particle and a stationary attractive Coulomb impurity ${ }^{10,14}$. For DSI to occur, we need the supercritical condition $a>1$ to occur in the system, where $a=Z e^{2} / \varepsilon \hbar v_{\mathrm{F}}$ is the effective fine-structure constant of the impurity. Here $Z$ is the ionicity, $\varepsilon$ is the background dielectric constant, and $v_{\mathrm{F}}$ is the Fermi velocity. When the supercritical condition holds, the radius of the quasi-bound states of the impurity is discrete scale invariant according to the relation $\frac{R_{n+1}}{R_{n}}=\sqrt{\lambda}=e^{\frac{\pi}{s_{0}}}$ with $s_{0}=\sqrt{a^{2}-1} \cdot R_{n}$ is the characteristic radius of the quasi-bound state and when the magnetic length $\ell_{n}=\sqrt{\frac{\hbar c}{e B_{n}}}=s_{0} R_{n}$ under an applied magnetic field, the energy of the $n$-th quasi-bound states rises rapidly and moves through the Fermi energy. Hence, the sequence of fields at which there is a peak in the density of states at the Fermi energy satisfies $\frac{B_{n+1}}{B_{n}}=e^{2 \pi / s_{0}}=e^{2 \pi / \sqrt{a^{2}-1}}=\lambda^{10}$. When $B=B_{n}$, the resonant scattering between the mobile carriers and the quasi-bound states influences the transport properties and results in a log-periodic correction to the MR and Hall traces.

The most interesting feature of our results is that compared to the bulk or thick flakes of $\mathrm{ZrTe}_{5}$ and $\mathrm{HfTe}_{5}$, the scale factor $\lambda$ in the thinner flakes becomes quite small. As mentioned above, $\lambda$ is a monotonically decreasing function of $a$ and $a=Z e^{2} / \varepsilon \hbar v_{\mathrm{F}}$. The Fermi velocity $v_{F}$ is expected to be the same in bulk and thin flakes of a Dirac material. The background dielectric constant $\varepsilon$ depends on the core electrons and so it is also unlikely to change. Thus, we must look for changes in $Z$ to explain the change in $\lambda$.

Based on Figs. 2-4, we notice that the ratio of oscillation amplitude to MR, i.e., $\Delta R_{\mathrm{xx}}(B) / R_{\mathrm{xx}}(B)$, for the $n=1$ peak in thick and thin flakes are $1.3 \%$ and $0.5 \%$, respectively. The larger surfacevolume ratio but weaker oscillations in thinner flakes may exclude the possibility that the log-periodicity arises from a surface effect. By estimating from the Hall traces (Supplementary Figs. 1, 6), the Dirac hole carrier density of the 1200-nm-thick $\mathrm{ZrTe}_{5}$ flake is $2.6 \times$ $10^{15} \mathrm{~cm}^{-3}$, very close to that of bulk $\mathrm{ZrTe}_{5}$ (ref. ${ }^{10}$ ), whereas the Dirac carrier density of the $137-\mathrm{nm}$-thick $\mathrm{ZrTe}_{5}$ flake is $9.9 \times 10^{16}$ $\mathrm{cm}^{-3}$. The greatly increased carrier density in the thinner flakes can have a profound effect on screening of the Coulomb interaction. There are three types of screening in the transitionmetal pentatelluride compounds, including the usual effects arising from bound electrons and conduction carriers, as well as vacuum polarization. The screening from the bound core electrons is responsible for background dielectric constant $\varepsilon$. As mentioned above, this screening effect is unlikely to change between the bulk and flakes since it depends only on very local features of the atomic structure. The screening from conduction carriers occurs in ordinary metals and in Weyl metals. It is noticed that the screening effect does not change the effective charge (ionicity $Z$ ) and has little influence on $\lambda$. The vacuum polarization is a specific feature of Dirac and Weyl semimetals and comes from the fact that the joint density of states for particle-hole excitations is large at low energies ${ }^{38,39}$. The introduction of an impurity charge excites many virtual particle-hole pairs in Dirac and Weyl semimetals, much more than in a typical insulator with a hard gap. In ref. ${ }^{38}$, it was found in two dimensions that this effect renormalizes $Z$ downward: $Z \rightarrow Z^{*}=Z$ / [1 $\left.+Z Q \ln (1 / \kappa a)\right]$, where $\kappa$ is proportional to the Fermi wavevector and thus to the square root of the carrier concentration $n_{\mathrm{e}} ; a$ is a short-distance cutoff at the atomic scale and $Q$ is a numerical factor of order 1 . A review of interaction effects in the Coulomb impurity problem in graphene concluded that this is the main effect ${ }^{40}$. There is no such complete theory in three dimensions to our knowledge, but the physics is expected to be similar. The renormalization of $Z$ is substantial due to its large starting value and indeed if $n_{\mathrm{e}} a^{2}$ is of order $10^{-4}$, as in our thin flakes, then changes in $\lambda$ by a factor of 2 are easily obtained. Essentially, in the thinner flakes the vacuum polarization effect decreases and the effective charge becomes larger, which gives an increase in $s_{0}$ and a smaller $\lambda$. Therefore, we propose that vacuum polarization plays a crucial role in the decrease of $\lambda$ in 
flakes. Further theoretical efforts are still needed to make this connection fully quantitative.

Although at $4.2 \mathrm{~K}$ there are more than three oscillating cycles detected in the thin flakes due to the smaller scale factor, the oscillation signal becomes clear only above 3-5T for the thin flakes, much higher than that for thick flakes or bulk ${ }^{10}$. We explain that the critical field $B_{c}$ over which the oscillations should be observed is closely related to the Thomas-Fermi screening length $\xi$. Though the screening from conduction carriers has little influence on $\lambda$, it can affect the visibility of the oscillations by converting the Coulomb potential $Z e^{2} / r$ to $\frac{Z e^{2}}{r} \times e^{-r / \xi}$. When the distance from the impurity is greater than $\xi$, the Coulomb potential becomes exponential and DSI is lost. In the thinner flakes, the increased carrier density leads to decreased $\xi$ accompanying with a larger $B_{c}$. For the thinner flakes, the calculated $B_{c} \sim 3.5 \mathrm{~T}$ and the number of oscillations $N \sim 5$ are consistent with the experimental results (see Supplementary Note 2 for more details).

In summary, we carried out systematic transport measurements on the transition-metal pentatelluride $\mathrm{ZrTe}_{5}$ and $\mathrm{HfTe}_{5}$ flakes with different thicknesses. LogB-periodic oscillations and DSI feature are detected in both the MR and the Hall traces of these flakes. The scale factor is observed to decrease with decreasing thickness, which is attributed to the weaker vacuum polarization effect resulting from higher carrier density. This work offers a way to tune the DSI and log-periodic oscillations in quantum materials. It also provides deep insights into many-body effects with a Diraclike spectrum.

\section{METHODS}

Sample information

The high-quality $\mathrm{ZrTe}_{5}$ and $\mathrm{HfTe}_{5}$ single crystals were grown by the Te-flux method as described in previous reports ${ }^{10,14,37}$. The crystals were characterized by powder X-ray diffraction, SEM with energy dispersive Xray spectroscopy, and transmission electron microscopy.

\section{Device fabrication}

The $\mathrm{ZrTe}_{5}$ and $\mathrm{HfTe}_{5}$ flakes were exfoliated by using the Scotch tape method onto 300-nm-thick $\mathrm{SiO}_{2} / \mathrm{Si}$ substrates. After spin coating of poly (methyl methacrylate) (PMMA), the standard electron beam lithography in a FEI Helios NanoLab 600i Dual Beam System was carried out to define electrodes. Metal electrodes $(\mathrm{Pd} / \mathrm{Au}, 6.5 / 300 \mathrm{~nm})$ were deposited in a LJUHV E-400L E-Beam Evaporator after Ar plasma cleaning.

\section{Transport measurements}

Electronic transport measurements in this work were mainly conducted in the pulsed high magnetic field facility (53 T) at Wuhan National High Magnetic Field Center. Standard six-electrode-method was used for the measurements. The current is applied along the $a$ axis and the magnetic field is along the $b$ axis for all measurements. Gold wires are attached to the electrodes by the silver epoxy and fixed on the substrate by GE varnish to avoid the vibration under pulsed field (see Supplementary Fig. 9).

\section{DATA AVAILABILITY}

The data that support the findings of this study are available from the corresponding author on reasonable request.

\section{CODE AVAILABILITY}

The code in this work is available from the corresponding author on reasonable request.

Received: 24 August 2020; Accepted: 9 November 2020; Published online: 04 December 2020

\section{REFERENCES}

1. Sornette, D. Discrete-scale invariance and complex dimensions. Phys. Rep. 297, 239-270 (1998).

2. Braaten, E. \& Hammer, H. W. Universality in few-body systems with large scattering length. Phys. Rep. 428, 259-390 (2006).

3. Efimov, V. Energy levels arising from resonant 2-body forces in a 3-body system. Phys. Lett. B 33, 563-564 (1970).

4. Kraemer, T. et al. Evidence for Efimov quantum states in an ultracold gas of caesium atoms. Nature 440, 315-318 (2006).

5. Huang, B., Sidorenkov, L. A., Grimm, R. \& Hutson, J. M. Observation of the second triatomic resonance in Efimov's scenario. Phys. Rev. Lett. 112, 190401 (2014).

6. Pires, R. et al. Observation of Efimov resonances in a mixture with extreme mass imbalance. Phys. Rev. Lett. 112, 250404 (2014).

7. Tung, S. K. et al. Geometric scaling of Efimov states in a ${ }^{6} \mathrm{Li}^{133} \mathrm{Cs}$ mixture. Phys. Rev. Lett. 113, 240402 (2014).

8. Shytov, A. V., Katsnelson, M. I. \& Levitov, L. S. Atomic collapse and quasi-Rydberg states in graphene. Phys. Rev. Lett. 99, 246802 (2007).

9. Nishida, Y. Vacuum polarization of graphene with a supercritical Coulomb impurity: low-energy universality and discrete scale invariance. Phys. Rev. B 90, 165414 (2014).

10. Wang, H. C. et al. Discovery of log-periodic oscillations in ultraquantum topological materials. Sci. Adv. 4, eaau5096 (2018).

11. Ovdat, $O$. et al. Observing a scale anomaly and a universal quantum phase transition in graphene. Nat. Commun. 8, 507 (2017).

12. Liu, $\mathrm{H}$. et al. Discrete scale invariance in topological semimetals. Preprint at https://arxiv.org/abs/1807.02459 (2018).

13. Zhang, P. \& Zhai, H. Efimov effect in Dirac semi-metals. Front. Phys. 13, 137204 (2018).

14. Wang, $H$. et al. Log-periodic quantum magneto-oscillations and discrete-scale invariance in topological material $\mathrm{HfTe}_{5}$. Natl Sci. Rev. 6, 914 (2019).

15. Zhang, N. et al. Magnetotransport signatures of Weyl physics and discrete scale invariance in the elemental semiconductor tellurium. Proc. Natl Acad. Sci. USA 117, 11337 (2020).

16. Weng, H. M., Dai, X. \& Fang, Z. Transition-metal pentatelluride $\mathrm{ZrTe}_{5}$ and $\mathrm{HfTe}_{5}$ : a paradigm for large-gap quantum spin Hall insulators. Phys. Rev. X 4, 011002 (2014).

17. Fan, Z. J. et al. Transition between strong and weak topological insulator in $\mathrm{ZrTe}_{5}$ and $\mathrm{HfTe}_{5}$. Sci. Rep. 7, 45667 (2017).

18. Shahi, P. et al. Bipolar conduction is the origin of the electronic transition in pentatellurides: metallic vs. semiconducting behavior. Phys. Rev. X 8, 021055 (2018).

19. Li, Q. et al. Chiral magnetic effect in ZrTes. Nat. Phys. 12, 550-554 (2016).

20. Shen, L. et al. Spectroscopic evidence for the gapless electronic structure in bulk $\mathrm{ZrTe}_{5}$. J. Electron Spectrosc. Relat. Phenom. 219, 45-52 (2017).

21. Chen, R. Y. et al. Magnetoinfrared spectroscopy of Landau levels and Zeeman splitting of three-dimensional massless Dirac fermions in $\mathrm{ZrTe}_{5}$. Phys. Rev. Lett. 115, 176404 (2015).

22. Zheng, G. L. et al. Transport evidence for the three-dimensional Dirac semimetal phase in $\mathrm{ZrTe}_{5}$. Phys. Rev. B 93, 115414 (2016).

23. Liang, T. et al. Anomalous Hall effect in $\mathrm{ZrTe}_{5}$. Nat. Phys. 14, 451-455 (2018).

24. Ge, J. et al. Unconventional Hall Effect induced by Berry Curvature. Natl. Sci. Rev. https://doi.org/10.1093/nsr/nwaa163 (2020).

25. Wu, R. et al. Evidence for topological edge states in a large energy gap near the step edges on the surface of $\mathrm{ZrTe}_{5}$. Phys. Rev. X 6, 021017 (2016).

26. Manzoni, G. et al. Evidence for a strong topological insulator phase in $\mathrm{ZrTe}_{5}$. Phys. Rev. Lett. 117, 237601 (2016).

27. Li, X.-B. et al. Experimental observation of topological edge states at the surface step edge of the topological insulator $\mathrm{ZrTe}_{5}$. Phys. Rev. Lett. 116, 176803 (2016).

28. Zhang, Y. et al. Electronic evidence of temperature-induced Lifshitz transition and topological nature in $\mathrm{ZrTe}_{5}$. Nat. Commun. 8, 15512 (2017)

29. Niu, J. J. et al. Electrical transport in nanothick $\mathrm{ZrTe}_{5}$ sheets: From three to two dimensions. Phys. Rev. B 95, 035420 (2017).

30. Zhou, Y. et al. Pressure-induced superconductivity in a three-dimensional topological material ZrTe 5 . Proc. Natl Acad. Sci. USA 113, 2904-2909 (2016).

31. Qi, Y. P. et al. Pressure-driven superconductivity in the transition-metal pentatelluride $\mathrm{HfTe}_{5}$. Phys. Rev. B 94, 054517 (2016).

32. Liu, Y. et al. Superconductivity in $\mathrm{HfTe}_{5}$ across weak to strong topological insulator transition induced via pressures. Sci. Rep. 7, 44367 (2017).

33. Zheng, G. L. et al. Field-induced topological phase transition from a threedimensional Weyl semimetal to a two-dimensional massive Dirac metal in $\mathrm{ZrTe}_{5}$. Phys. Rev. B 96, 121401(R) (2017).

34. Liu, Y. et al. Zeeman splitting and dynamical mass generation in Dirac semimetal $\mathrm{ZrTe}_{5}$. Nat. Commun. 7, 12516 (2016). 
35. Tang, F. et al. Three-dimensional quantum Hall effect and metal-insulator transition in $\mathrm{ZrTe}_{5}$. Nature 569, 537-541 (2019).

36. Okada, S., Sambongi, T. \& Ido, M. Giant resistivity anomaly in $\mathrm{ZrTe}_{5}$. J. Phys. Soc. Jpn. 49, 839-840 (1980).

37. Wang, H. C. et al. Chiral anomaly and ultrahigh mobility in crystalline $\mathrm{HfTe}_{5}$. Phys. Rev. B 93, 165127 (2016)

38. Katsnelson, M. I. Nonlinear screening of charge impurities in graphene. Phys. Rev. $B$ 74, 201401(R) (2006).

39. Shytov, A. V., Katsnelson, M. I. \& Levitov, L. S. Vacuum polarization and screening of supercritical impurities in graphene. Phys. Rev. Lett. 99, 236801 (2007).

40. Kotov, V. N. et al. Electron-electron interactions in graphene: current status and perspectives. Rev. Mod. Phys. 84, 1067 (2012).

\section{ACKNOWLEDGEMENTS}

We thank Liqin Huang, Zihan Yan, Qingzheng Qiu, Haiwen Liu, and Yongjie Liu for discussions on the data. This work was financially supported by the National Key Research and Development Program of China (2018YFA0305604 and 2017YFA0303302), the National Natural Science Foundation of China (Grant Nos. 11888101, 11774008, 12004441), Beijing Natural Science Foundation (Z180010), and the Strategic Priority Research Program of Chinese Academy of Sciences (Grant No. XDB28000000). J.Y. and D. M. were supported by the U.S. Department of Energy, Office of Science, Basic Energy Sciences, Materials Sciences and Engineering Division. H.W. acknowledges the support of the Hundreds of Talents program of Sun Yat-Sen University and the Fundamental Research Funds for the Central Universities (No. 20lgpy165).

\section{AUTHOR CONTRIBUTIONS}

Jian Wang conceived and supervised the research. Y.Liu, H.W. performed the transport measurements. Y.Li and J.G. fabricated the devices. J.Y. and D.M. grew the $\mathrm{ZrTe}_{5}$ and $\mathrm{HfTe}_{5}$ bulk crystals. H.Z., Junfeng Wang, L.L., and J.-Y.D. helped in the transport measurements. R. J. carried out theoretical calculations. Y.Liu, H.W., and Jian Wang analyzed the data. Y.Liu, H.W., R.J., and Jian Wang wrote the manuscript with input from all authors.

\section{COMPETING INTERESTS}

The authors declare no competing interests.

\section{ADDITIONAL INFORMATION}

Supplementary information is available for this paper at https://doi.org/10.1038/ s41535-020-00290-6.

Correspondence and requests for materials should be addressed to J.W.

Reprints and permission information is available at http://www.nature.com/ reprints

Publisher's note Springer Nature remains neutral with regard to jurisdictional claims in published maps and institutional affiliations.

Open Access This article is licensed under a Creative Commons adaptation, distribution and reproduction in any medium or format, as long as you give appropriate credit to the original author(s) and the source, provide a link to the Creative Commons license, and indicate if changes were made. The images or other third party material in this article are included in the article's Creative Commons license, unless indicated otherwise in a credit line to the material. If material is not included in the article's Creative Commons license and your intended use is not permitted by statutory regulation or exceeds the permitted use, you will need to obtain permission directly from the copyright holder. To view a copy of this license, visit http://creativecommons. org/licenses/by/4.0/.

(c) The Author(s) 2020 